\title{
Endoscopic Endonasal Approach in Clival Chordoma Surgery: Case Series
}

\author{
Chien Ying Vincent $\mathrm{Ngu}^{1} \cdot$ Ing Ping Tang ${ }^{2}\left(\mathbb{D} \cdot\right.$ Boon Han Kevin $\mathrm{Ng}^{2} \cdot$ \\ Albert S. I. I. Hieng Wong ${ }^{3}$ - Donald Ngian San Liew ${ }^{3}$
}

Received: 10 December 2020/Accepted: 8 February 2021 / Published online: 23 February 2021

(C) Association of Otolaryngologists of India 2021

\begin{abstract}
Chordomas are rare and slow-growing locally destructive bone tumors that can develop in the craniospinal axis. It is commonly found in the sacrococcygeal region whereas only $25-35 \%$ are found in the clival region. Headache with neurological deficits are the most common clinical presentations. Complete surgical resection either via open or endoscopic endonasal approaches are the main mode of treatment. Here, we report a series of 5 cases of clival chordomas which was managed via endoscopic endonasal approaches in our center. A retrospective analysis of patients who had undergone endoscopic endonasal resection of clival chordoma in Sarawak General Hospital from 2014 to 2018. A total of 5 cases were operated on endoscopically via a combine effort of both the otorhinolaryngology team and the neurosurgical team during the study period from year 2013 to 2018. From our patient, 2 were female and 3 were male patients. The main clinical presentation was headache, squinting of eye and nasopharyngeal fullness. All our patient had endoscopic endonasal debulking of clival tumor done, with average of hospital stay from $9-23$ days. Pos-operatively, patients were discharged back well. Endoscopic endonasal resection of
\end{abstract}

Ing Ping Tang

iptang@unimas.my

1 Department of Otorhinolaryngology, Sarawak General Hospital, Otorhinolaryngology - Head and Neck Surgery Department, Faculty of Medicine, University of Malaya, Kuala Lumpur, Malaysia

2 Department of Otorhinolaryngology, Sarawak General Hospital, Department of ORL-HNS, Faculty of Medicine and Health Sciences, University Malaysia Sarawak, 94300 Kota Samarahan, Sarawak, Malaysia

3 Department of Neurosurgery, Sarawak General Hospital, Kuching, Malaysia clival chordomas gives good surgical resection results with low morbidity rates and therefore can be considered as a surgical option in centers where the surgical specialties are available.

Keywords Endoscopic endonasal $\cdot$ Clival $\cdot$ Chordoma

\section{Introduction}

Chordomas are rare and slow growing locally destructive bone tumor with an incidence rate of 0.08 in 100,000 of population $[1,2]$. It is more commonly found at the scarococygeal region (50-60\%), followed by the spheno-occipital region (25-35\%) and the vertebral column (10\%) [3]. Chordomas have a higher incidence in male compared to female [1].

Clival chordomas arises from fetal/ embryonal notochord remnants, that usually forms the nucleus pulposus of intervertebral discs, whereas, in the cephalic end of notochord will differentiate into the precursor for formation of sella and the posterior body of sphenoid, and basiocciput bone [4-6]. This malignant change usually occur between ages 50-60 years old, with low incident rate before 40 years old [7]. Its occurrence in children will present in aggressive pattern. The main aim of treatment is surgical resection. Patients with complete tumor resection has better prognosis. 